\title{
CORRIGENDUM
}

doi:10.1038/nature09384

\section{Detection of functional haematopoietic stem cell niche using real-time imaging}

Yucai Xie ${ }^{1,2}$, Tong Yin ${ }^{2}$, Winfried Wiegraebe ${ }^{2}, \mathrm{XiC}$. $\mathrm{He}^{2}$, Diana Miller ${ }^{3}$, Danny Stark ${ }^{2}$, Katherine Perko ${ }^{2}$, Richard Alexander ${ }^{2}$, Joel Schwartz'2, Justin Grindley², Jungeun Park'2, Jeff Haug', Joshua Wunderlich ${ }^{2}$, Hua $\mathrm{Li}^{2}$, Simon Zhang ${ }^{2}$, Teri Johnson ${ }^{2}$, Ricardo A. Feldman ${ }^{3}$ \& Linheng $\mathrm{Li}^{2,4}$

${ }^{1}$ Department of Cardiology, Shanghai Rui Jin Hospital, Shanghai Jiao Tong University School of Medicine, 197, Rui Jin 2 Road, Shanghai 200025, China. ${ }^{2}$ Stowers Institute for Medical Research, 1000 E. 50th Street, Kansas City, Missouri 64110, USA. ${ }^{3}$ Department of Microbiology and Immunology, and Greenebaum Cancer Center, University of

Maryland School of Medicine, Baltimore, Maryland 21201, USA. ${ }^{4}$ Department of

Pathology and Laboratory Medicine, Kansas University Medical Center, 3901 Rainbow Boulevard, Kansas City, Kansas 66160, USA.

\section{Nature 457, 97-101 (2009)}

In this Letter, affiliations 1 and 2 were in the wrong order. The correct affiliations are as shown here. 